\title{
La participación del alumnado en la vida del centro: una aproximación desde la comunidad educativa ${ }^{1}$
}

\section{Pupil participation in school life: an approach from the educational community}

\author{
Jordi L. COIDURAS RODRÍGUEZ ${ }^{1}$, M. Àngels BALSELLS BAILÓN ${ }^{1}$, Carles \\ ALSINET MORA $^{1}$, Aida URREA MONCLÚS ${ }^{1}$, Ignacio GUADIX GARCÍA ${ }^{2}$ \\ y Oscar BELMONTE CASTELL ${ }^{2}$ \\ ${ }^{1}$ Universidad de Lleida y ${ }^{2}$ UNICEF Comité Español
}

Recibido: Septiembre 2014

Evaluado: Diciembre 2014

Aceptado: Diciembre 2014

\section{Resumen}

La Convención de los Derechos del Niño (CDN, 1989) es actualmente el tratado internacional más ratificado. Distintos autores han puesto de relieve su potencialidad para una educación moral y en la ciudadanía. Aun así, paradójicamente, distintas investigaciones informan de su incorporación escasa u ocasional en las prácticas escolares. En este artículo se realiza una aproximación a las experiencias escolares sobre participación, la tercera $P$ de la CDN, desde la pluralidad de voces y actores de la comunidad educativa, mediante 14 grupos de discusión en 11 comunidades autónomas del estado español. El análisis de los discursos evidencia bajos niveles de participación del alumnado en la vida escolar. Pero, a la vez, se constata un escenario educativo propicio para el desarrollo de proyectos que contribuyan a la participación infantil, así como a la incorporación de la CDN como elemento motor de la vida del centro y referente integrador de los distintos proyectos de centro.

Palabras clave: participación infantil, experiencias escolares, Convención de los Derechos del Niño, ciudadanía.

\begin{abstract}
The Convention on the Rights of the Child (CRC, 1989) is currently the most ratified international treaty. Several authors have highlighted its potential for both a moral education and citizenship. However, paradoxically, different studies report its limited or occasional incorporation into school practices. This article explores experiences of participation in schools,
\end{abstract}

\footnotetext{
${ }^{1}$ Esta investigación ha sido posible gracias a la financiación de la Agencia Española de Cooperación Internacional para el Desarrollo (AECID) y de UNICEF - Comité Español, en el marco del proyecto "Fomento de la Educación para el Desarrollo en la Educación Formal" 2010 - 2012. El contenido de esta publicación es responsabilidad exclusiva de los autores y no refleja necesariamente la opinión de la AECID.
} 
the third $P$ of the CRC, from the plurality of voices and actors of the educational community, by means of 14 discussion groups in 11 autonomous communities in Spain. Discourse analysis evidence low levels of student participation in school life. But, at the same time, a favorable educational environment for the development of projects that contribute to child participation is found, as well as for the incorporation of the CRC as a mover and a referential integrator of the different schools projects. However, it is also an educational background conductive to projects for its development, such as the incorporation of the $\mathrm{CRC}$ as a referential integrator of the schools projects.

Keywords: child participation, school experiences, Convention on the Rights of the Child, citizenship.

La escuela como agente socializador asume un protagonismo particular en la inserción y participación del alumnado en la comunidad. Todo lo que en ella sucede concierne a la adquisición de valores, competencias, normas y significados culturales. En definitiva, facilita la experimentación de la pertinencia social en procesos de convivencia mediante la actividad con los demás. La participación e implicación del alumnado en esta realidad es una experiencia substantiva como aprendizaje para el ejercicio de la ciudadanía y por ello no debe postergarse. Parafraseando a Mayor Zaragoza (1989) en “mañana siempre es tarde”, las niñas y los niños como sujetos de derechos deben experimentarlos de forma objetiva y consciente en la escuela. Esto supone renunciar a lo que se ha llamado la "ideología de la inmadurez" y la visión adultocéntrica, a partir de la cual seguimos pensando recursos para la infancia obviando su parecer (Pulido Valero, Calderón López, Martín-Seoane, \& Lucas-Molina, 2014; Rudduck \& Flutter, 2007; Rudduck, 2007).

Con la aprobación de la Convención de los Derechos del Niño (CDN) por la asamblea de las Naciones Unidas (1989), se impulsa un movimiento a favor de la consideración de las niñas y los niños como miembros activos de la sociedad (Alsinet, 2000; Fielding, 2012; Veenhoven, 1996). En su condición de ciudadanas y ciudadanos, se reconocen derechos civiles reservados con anterioridad a los adultos: libertad de expresión y opinión; buscar, recibir y difundir información; pensamiento, conciencia y religión; libertad de asociación y de protección de su vida privada. A pesar del amplio acuerdo que suscita la CDN, distintas revisiones e investigaciones (Casas et al., 2008; Ceballos \& Susinos, 2012; Cots, 2005; Fuentes-Peláez, 2011; Trilla \& Novella, 2011) han puesto en evidencia la baja aplicación real de los derechos que agrupamos bajo la denominada tercera $P$, de participación. De hecho, encontramos una realidad débil y anecdótica en experiencias de implicación de la infancia en la toma de decisiones, en las propuestas escolares que se desarrollan y en la vida del centro (Fielding, 2004; Lodge, 2005; Mitra, Serriere, \& Kirshner, 2013).

UNICEF está impulsando diferentes experiencias para fomentar la participación infantil en los centros escolares. Estas experiencias pretenden aportar a los centros educativos los instrumentos pedagógicos necesarios para incorporar la CDN, haciendo de la participación del alumnado una finalidad educativa. La potencialidad educativa de la CDN es indudable como referente de análisis e interpretación de la realidad, pero también como código para vivir la alteridad en el reconocimiento de la pluralidad del 
pensamiento y de expresión. Es posible encontrar en la CDN una forma de integración entre experiencia de la democracia y la educación moral. La educación en derechos, la corresponsabilización con la comunidad y el compromiso con el propio aprendizaje son tres elementos relacionados con "vivir la CDN", que pueden reportar los beneficios que se derivan de una educación en la cultura moral que ya planteaban Durkheim, (1961) o Kohlberg, Power y Higgins (1997) entre otros. En esta línea consideramos que la educación es incompleta si no incorpora el conjunto de valores democráticos propios de la ciudadanía activa.

Un ejemplo de estas experiencias es el programa anglosajón Rights Respecting Schools Award (RRSA) (UNICEF, 2008) que provee un marco de actuación alrededor de cuatro grandes ejes: (1) liderazgo y guía hacia la integración de los valores de la CDN en la vida escolar; (2) conocimiento y comprensión de la CDN; (3) ambiente y cultura del aula; y (4) participación activa de los alumnos en la toma de decisiones del centro escolar. La experiencia de RRSA aporta resultados efectivos en el alumnado no solamente en el conocimiento de sus derechos, también en la comprensión y apropiación de las responsabilidades relacionadas. Para los impulsores de este programa el alumnado mejoró de forma significativa en: la distinción de derechos y "deseos"; en la necesidad de respetar los derechos de los demás y, a la vez, se estableció eficazmente la vinculación de la dimensión organizativa con la curricular, impregnándose mutuamente.

El interés por la contribución del alumnado a esta organización y, particularmente, por su potencialidad formativa en la adquisición de valores y el aprendizaje de la participación están en el origen de este texto.

\section{La tercera $P$ como experiencia escolar}

La experiencia de la participación derivada de nuestra legislación educativa supuso un cambio relevante con la Ley Orgánica Reguladoras del Derecho a la Educación (Ley Número 3, 1985) con la consideración del alumnado bien como miembros de los Consejos Escolares, junto a los representantes de las familias, del municipio y del personal de administración y servicios o bien, como miembros de la comunidad susceptibles de ser consultados en la regulación de la vida y los proyectos de centro. La fuerza de este tipo de proyectos reside en la relación de una concepción amplia de los resultados escolares, con la innovación centrada en la mejora de los procesos del aula y la promoción de la capacidad interna de cambio en cada centro escolar (Bolívar, 1999). La participación como propuesta de desarrollo institucional no puede verse, por tanto, de forma aislada; parece necesario relacionarla con la mejora de las experiencias educativas del alumnado (desarrollo curricular), la capacitación profesional en una comunidad formativa (desarrollo profesional) (Fullan, 1993) y la implicación de y con la comunidad (West y Hopkins, 2003). 


\section{Grado de la participación}

La participación representativa no contradice otras dinámicas para incrementar la voz del alumnado. Distintos autores se han ocupado de los grados de participación. Además de la originaria propuesta de Hart (1992) con su escala de ocho niveles - entre dos polos de participación simbólica y de participación genuina - encontramos otras gradaciones en base a la intensidad en el protagonismo, responsabilidad, compromiso, grado real de participación e implicación del alumnado en la toma de decisiones, en la organización del centro escolar y en el desarrollo de las actividades (Brown, 2001; Fielding y McGregor, 2005; Fielding, 2001; Martínez Rodríguez, 2010; Shier, 2001).

Susinos y Ceballos (2012) proponen una síntesis representando en un extremo la ausencia de participación y, en el otro, la máxima participación como aprendizaje intergeneracional. Al primer nivel, no considerado de participación o simplemente como participación simbólica, le seguirían otros tres niveles: (a) los alumnos como fuente de información, (b) los alumnos como coinvestigadores y, como mayor grado de participación, (c) el aprendizaje intergeneracional. En tanto que la opinión de los alumnos sobre aspectos determinados es tenida en cuenta (Martín del Pozo \& De Juanas Oliva, 2013), pueden concretarse acciones para una participación más vivida, adquiriendo su punto de vista significado y reconocimiento como forma de implicación. Cuando el alumnado incrementa su protagonismo en el diseño y desarrollo de proyectos, con mayor o menor ayuda docente, repercutiendo directamente en la organización o el currículum, reconocemos un segundo nivel de participación. Las alumnas y los alumnos, entonces, son considerados creadores de saber y coinvestigadores (Fielding, 2011). En el último y tercer nivel, de grado máximo de participación, el "aprendizaje intergeneracional como democracia vivida" se entiende como la forma usual de gestión del centro con el aprendizaje mutuo de alumnos y adultos, donde los procesos permiten la toma de decisiones relacionadas con las dimensiones organizativa y curricular. Cada uno de estos tres niveles tiene entidad propia, y a la vez queda incluido en el posterior por suponer un grado superior de implicación, protagonismo y poder del alumnado.

\section{Objetos de participación}

La participación de la infancia en los centros educativos suele tener dos elementos diferentes: participación en la vía curricular y participación en la vida organizativa. Susinos y Ceballos (2012), considerando dos grandes elementos de participación y sintetizando propuestas anteriores (Fielding \& Bragg, 2003; Rudduck \& Flutter, 2007; Smyth, 2007), proponen cinco grandes objetos de participación: la mejora de la organización y la gestión educativas, la negociación del currículo escolar, los cambios en el entorno físico y social de la escuela, la mejora docente y la intervención en la comunidad.

La organización y gestión del Centro es un cometido principal del Consejo Escolar (CE) con la implicación y representación de la comunidad educativa. Sin embargo, la participación e influencia real del alumnado es bastante limitada en las decisiones que importan al centro. Por otro lado, la ley no contempla hasta segundo curso de 
Educación Secundaria Obligatoria su representación en el CE, con lo que las experiencias a este nivel no abarcan todas las edades. Sin embargo, sí se visualiza más claramente el papel de la participación en la organización cuando se interpreta la comunidad educativa como: un espacio plural con diversidad de edades y de culturas, como un lugar donde se experimenta y argumenta la regulación de usos de los servicios $\mathrm{y}$ recursos, donde se reconocen los derechos y responsabilidades, o como una comunidad donde se experimentan la suma de contribuciones en la actividad común. Todo ello justifica las posibilidades de la organización y gestión escolar como espacio de implicación del alumnado, de diálogo y experiencia como línea de desarrollo organizacional. Así, como la necesidad de explorar nuevas vías para facilitar que la voz de los alumnos implique procesos de decisión traducibles en acciones como realizaciones concretas.

Las vivencias de negociación curricular, se relacionan con la selección de temáticas relacionadas con la vida (Hargreaves, Earl, y Ryan, 1998), para conectar con los intereses, preocupaciones y experiencias de los alumnos. Si el aprendizaje del currículum tiene como objetivo la socialización, parece oportuno considerar los objetos culturales y la realidad del centro escolar en lo que suponen de reto intelectual para conocer y comprender su contexto. Desde la perspectiva crítica del currículum (Connell, 1997; Torres Santomé, 2010) constatamos las posibilidades del currículum en su potencialidad socializadora, con un acercamiento a él desde la pertinencia a la realidad del alumnado.

Los cambios en el entorno físico y social de la escuela, se refieren a la investigación sobre la realidad cercana, la misma escuela y su contexto mediato e inmediato como otra vía de implicación en la mejora de los usos de los espacios o del funcionamiento de los servicios. El análisis del entorno permite su apropiación y un cambio argumentado en la búsqueda de innovaciones eficaces en los usos.

En las iniciativas de mejora docente, algunas experiencias (Fielding \& Bragg, 2003; Rudduck \& Flutter, 2007) sitúan al alumno en el diálogo y crítica pedagógica, en la definición del buen docente, desde su visión sobre cuáles deben ser sus competencias, sus características en la conducción del grupo o de la buena lección y la propuesta metodológica. En las experiencias de equipos docentes como comunidad participativa, éstas se consideran valiosas en cuanto aportan una visión de la acción docente desde las vivencias de los destinatarios (Jiménez-Trens \& Navaridas, 2012).

La intervención en la comunidad, en el barrio o en el municipio puede ser motivada y acompañada desde la escuela, pero su dimensión trasciende el escenario escolar. Entre estas experiencias los Consejos Infantiles nos interesan por reconocer en la voz de las niñas y los niños como ciudadanos su experiencia, opinión y propuestas para una ciudad más adecuada a sus necesidades, como propone Tonucci (1998) y la red de Ciudades Amigas de la Infancia promovida por UNICEF.

\section{Los escenarios, el nivel y el formato de la participación}

Las experiencias de participación encuentran en la escuela, según la población de referencia, distintos escenarios de acción. Desde el aula a una orientación más comunitaria de ciudad, pasando por el ciclo y el centro en su globalidad, pueden 
catalogarse distintas prácticas de implicación y voz del alumnado. El aula puede ser un espacio de procesos proactivos en la toma de decisiones que permiten desarrollar capacidades de reflexión y elección. El centro educativo, como escenario social más amplio presenta situaciones susceptibles para el desarrollo de la cohesión y la mediación en la resolución de conflictos, de un clima educativo propicio al aprendizaje y la convivencia, de escucha activa y de diálogo entre todos los miembros de la comunidad. En el entorno, los niños, niñas y adolescentes deben considerarse ciudadanos de presente, miembros activos de la comunidad donde exponen sus proyectos, sus opiniones y cumplen con sus obligaciones y sus responsabilidades.

El nivel educativo, como momento evolutivo, supone una adecuación de planteamiento de la actividad, considerando la lógica interna, la complejidad de la actividad como tarea cognitiva y en su grado de abstracción.

Otra de las dimensiones estudiadas en relación a la participación es el formato que ésta adopta. El más común suele ser el oral, en menor medida el escrito, a los que habría que sumar la representación visual o plástica para incorporar voces que a menudo permanecen en silencio (Greene, 2005). Creemos interesante considerar un formato de acción o enactivo (Bruner, 2013) en el que contemplamos la participación que implica el hacer, como uno de los formatos preferidos en las etapas de educación infantil, primaria y secundaria. $\mathrm{Y}$ un formato digital o multilenguaje que integra distintas formas de expresión, comunicación, colaboración y construcción.

De todo esto se desprende una diversidad de focos para fomentar la participación infantil en las instituciones educativas, así como los beneficios en el desarrollo de la infancia cuando se hace "vivir" la participación como finalidad educativa.

\section{Planteamiento metodológico}

UNICEF-Comité Español, plantea como reto aportar elementos pedagógicos que ayuden a los centros educativos a incorporar la CDN y la participación en sus organizaciones. Con esta finalidad, se proyecta una investigación para analizar la realidad española y ajustar las propuestas a las posibilidades en cada situación pedagógica.

Como objetivos principales se propuso:

(1) conocer y analizar las experiencias de participación del alumnado e

(2) identificar las barreras de la organización escolar para fomentarla.

Nos interesamos por las experiencias como vivencias, más allá de lo conceptual, facilitadoras de la apropiación de la CDN. Se trata de una vía diagnóstica útil para la generación de propuestas orientadas a reducir las discrepancias en relación a nuestras aspiraciones sobre la infancia (Balsells, 2011; Balsells, Coiduras, Alsinet, y Urrea, 2012).

El estudio que presentamos es una investigación de carácter descriptivo exploratorio llevada a cabo en España entre los años 2011-2012. La técnica elegida 
para la obtención de información fue el focus group por su potencialidad en la identificación de patrones para explicar, a partir de los actores, la realidad sobre la participación en el centro escolar. Todos los miembros de la comunidad educativa han participado como informadores y sus aportaciones han sido de crucial importancia ya que han permitido contrastar las necesidades de todos ellos: profesorado, alumnado, padres y madres y otros miembros de la comunidad.

\section{Participantes}

Se desarrollaron 14 grupos de discusión (GD) en las comunidades autónomas (CCAA) de Andalucía, Aragón, Asturias, Baleares, Canarias, Castilla - La Mancha, Cataluña, Galicia, Madrid, Navarra y País Vasco con una participación de 124 personas entre docentes, madres y padres, alumnado de educación primaria y secundaria obligatoria, así como otros miembros de la comunidad escolar (Tabla 1). Los informantes fueron seleccionados desde el criterio de la diversidad para poder documentar la variabilidad, con sus diferencias, coincidencias y particularidades (Blanchet y Gotman, 2005). Con Kaufmann (2011) situamos el acento, más que en la constitución de la muestra, en la buena elección de los informadores: la relación con las etapas de educación obligatoria de primaria, secundaria (escuelas, colegios, institutos y centros de educación especial); la pertenencia $(42,85 \%)$ y no pertenencia $(57,15 \%)$ a la red de escuelas ENRÉDATE de UNICEF y la condición de centros concertados, privados y públicos. Además se incorporan a los grupos docentes con responsabilidades de gestión o de coordinación pedagógica en el centro educativo.

\begin{tabular}{cccccc}
\hline $\begin{array}{c}\text { Papel en la } \\
\text { comunidad } \\
\text { educativa }\end{array}$ & $\begin{array}{c}\text { Docentes } \\
\text { Educación } \\
\text { Primaria }\end{array}$ & $\begin{array}{c}\text { Docentes } \\
\text { Educación } \\
\text { Secundaria }\end{array}$ & $\begin{array}{c}\text { Madres y } \\
\text { Padres }\end{array}$ & $\begin{array}{c}\text { Alumnado } \\
\text { Ed. Primaria } \\
\text { y Secundaria }\end{array}$ & $\begin{array}{c}\text { Personal de } \\
\text { Administració } \\
\text { n y Servicios }\end{array}$ \\
\hline $\mathbf{N}^{\mathbf{0}}$ & 49 & 20 & 9 & 45 & 1 \\
$\%$ & 39,52 & 16,13 & 7,25 & 36,29 & 0,81 \\
\hline
\end{tabular}

Tabla 1: Participantes a los Grupos de Discusión

\section{Instrumentos, procedimiento y análisis}

La formación de los grupos, el cronograma y su desarrollo se acordaron entre el equipo de investigación y el Comité Español de UNICEF, con el que: (a) se concretaron los contenidos y objetivos de la investigación; (b) se establecieron las líneas generales para la elaboración de los guiones y (c) la forma de acceso, organización y comunicación con los técnicos de Educación para el Desarrollo de UNICEF que los conducirían en las respectivas comunidades autónomas. Se configuraron 7 tipologías de grupos (Tabla 2). Para un desarrollo de los grupos similar en las 11 CCAA se elaboró el guión de preguntas, una ficha para los participantes de datos sociodemográficos y una guía básica de directrices para la conducción y gestión de las dinámicas de focus group. 


\begin{tabular}{|c|c|c|}
\hline Tipologías & Perfiles & Comunidad \\
\hline Consejo Escolar & Padres, profesores, alumnos, PAS del Consejo Escolar & $\begin{array}{l}\text { Andalucía } \\
\text { Navarra }\end{array}$ \\
\hline Alumnos & $\begin{array}{l}\text { Plenario Municipal de Infancia } \\
\text { Plenario Municipal de Infancia } \\
\text { Alumnado Centro Escolar }\end{array}$ & $\begin{array}{l}\text { Asturias I } \\
\text { Asturias II } \\
\text { Canarias }\end{array}$ \\
\hline $\begin{array}{l}\text { Profesorado } \\
\text { Primaria }\end{array}$ & $\begin{array}{l}\text { Docentes de Ed. Primaria: del equipo directivo y sin carga } \\
\text { directiva; miembros "escuelas enredadas" y de "escuelas } \\
\text { no enredada" }\end{array}$ & $\begin{array}{l}\text { Canarias } \\
\text { Aragón } \\
\text { Castilla-La Mancha } \\
\text { Galicia }\end{array}$ \\
\hline $\begin{array}{l}\text { Profesorado } \\
\text { Secundaria }\end{array}$ & $\begin{array}{l}\text { Docentes de Ed. Secundaria: del equipo directivo y sin } \\
\text { carga directiva; miembros "escuelas enredada" y de } \\
\text { "escuelas no enredada" }\end{array}$ & Cataluña I \\
\hline $\begin{array}{l}\text { Centros } \\
\text { "enredados" }\end{array}$ & Docentes de "escuelas enredadas" & Madrid \\
\hline $\begin{array}{l}\text { Centros "no } \\
\text { enredados" }\end{array}$ & Docentes de "escuelas no enredadas" & $\begin{array}{l}\text { País Vasco } \\
\text { Baleares } \\
\end{array}$ \\
\hline $\begin{array}{l}\text { Centros } \\
\text { Especiales }\end{array}$ & Docentes de centros con alta diversidad & Cataluña II \\
\hline
\end{tabular}

Tabla 2. Tipología de grupos

Las sesiones fueron grabadas en audio y transcritas de forma literal. Mediante el programa Atlas-ti v.5.0, software de Análisis Cualitativo de Datos, se creó la Hermenéutic Unit Editor en la que se incluyeron los datos (primary documents), los códigos y las anotaciones textuales (memos) realizadas en el proceso de análisis. En el análisis de contenido se localizó la información esencial contenida en los discursos, mediante un análisis temático consistente "[...] en leer del corpus, fragmento por fragmento, para definir el contenido codificándolo" (Fallery y Rodhain, 2007, p. 20) con una orientación bottom-up seleccionando en la primera etapa los párrafos, fragmentos y citas significativas dentro de los documentos de transcripción.

Las categorías, derivadas de la revisión bibliográfica, permitieron en una segunda etapa, la delimitación de subcategorías emergentes del análisis de los textos (Tabla 3) para relacionarlas posteriormente unas con otras y con la literatura científica. Ambas etapas se realizaron por dobles jueces del equipo de investigación y en distintas veces hasta llegar a la saturación. 


\begin{tabular}{ll}
\hline \multicolumn{1}{c}{ Categorías } & \multicolumn{1}{c}{ Subcategorías } \\
\hline a. Objeto de la participación & a.1.- Mejora de la organización \\
& a.2.- negociación del currículum \\
& a.3.- intervención en la comunidad \\
\hline b. Escenario & b.1.- aula \\
& b.2.- centro \\
& b.3.- comunidad \\
\hline c. Grado de participación & c.1.- fuente de información y consulta \\
& c.2.- participantes activos \\
\hline
\end{tabular}

d. Formato

Tabla 3: Sistema de categorías y subcategorías

\section{Análisis e interpretación de los resultados}

En este apartado cruzamos el análisis de los textos, en base a las categorías, con los dos objetivos de la investigación: (1) conocer y analizar las experiencias de participación del alumnado e (2) identificar las barreras de la organización escolar para fomentarla.

La presencia de la tercera $P$ en los discursos de los grupos de discusión realizados es escasa. El mismo concepto de participación aparece de forma diversa, asociándola a los distintos grados en que puede mostrarse, pero básicamente centrado en acciones que no suponen una participación real, sino más bien decorativa o simbólica (Hart, 1992). El conocimiento profesional de los derechos de la CDN vinculados a la participación se queda en la superficie de su verdadero sentido. Las intervenciones muestran la falta de un consenso básico sobre el significado profundo del concepto, de acuerdo con las apreciaciones de Susinos y Ceballos (2012). Un docente lo expresó con la metáfora de un árbol, de la siguiente forma:

“[...] Evidentemente los derechos tienen un sujeto, los derechos no están en el aire, los derechos también tienen un tronco al cual se amarran, que es la persona [...] creo que los centros y el sistema educativo está olvidando que detrás de mil asignaturas, organigramas, inventos y estructuras. Debería haber un proyecto de persona." GD de Profesionales de la Educación

¿Cuál es el objeto de las experiencias de participación infantil en los centros escolares?

Algunas experiencias de mejora de la organización muestran la implicación de toda la comunidad en la elaboración de documentos como respuesta a necesidades concretas. Suele evidenciarse una relación implícita con el Proyecto Educativo de Centro, con los grandes objetivos y principios que la comunidad se propone alcanzar. En los grupos de discusión se presentan experiencias donde de implicación del alumnado para la construcción de referentes de regulación de la convivencia: 
"Entre todos, las familias, los alumnos y los profesores, [...] se pidió la opinión y se elaboró una carta de convivencia. [...] se redactaron unos derechos y unos deberes como si fuéramos una población." GD de Profesionales de la Educación

La regulación de las relaciones y conductas es una de las temáticas reiterativas en las experiencias de participación. En opinión de los docentes hay una conciencia creciente sobre la relación entre el clima humano en el centro escolar y la educación en valores. Sin embargo, se constata un planteamiento de carácter reactivo en respuesta a los sucesos del día a día. No se plantea la implicación como propuestas de tipo anticipatorio y preventivo, por ejemplo, para la regulación de la convivencia. La literatura nos ha mostrado como algunas prácticas formativas para la resolución de conflictos con la implicación del alumnado, madres y padres y docentes con esa orientación proactiva han resultado eficaces. Las experiencias británicas recogidas en RRSA (Sebba y Robinson, 2010) presentan como el incremento en la participación de niñas y niños supone la mejora de las relaciones y la reducción de comportamientos de intimidación; el desarrollo de actitudes positivas hacia la diversidad y la disminución de prejuicios; así como el incremento de la implicación e identificación con el centro escolar.

Se ha detectado una ausencia relevante de planificación de actividades participativas en negociación del currículum; la principal limitación manifestada es de carácter organizacional: la presión sobre los resultados justifica la falta de iniciativas. Desde esta visión se desvinculan los procesos educativos en las competencias básicas de la experiencia de la participación o bien, se manifiesta un pensamiento que los situaría en un plano secundario.

"Muchos profesores no dejan a sus alumnos participar como deberían, pues por la amplitud de temarios que hay que dar, les es más fácil imponer el ritmo de trabajo y otras pautas, que dejarles decidir" GD de Profesionales de la Educación

En cambio, proponemos una visión de la participación presente en toda la vida escolar y prácticas educativas, en la "trama de la vida colectiva" (Rodríguez Sedano y Aguilera, 2009). Las exigencias administrativas actuarían de barrera en el proyecto de formación ética y de derechos. Estas exigencias, bajo una apariencia de neutralidad técnica, resultan casi siempre incompatibles con un planteamiento de educación para la participación y la ciudadanía. De forma implícita la falta de impulso y baja presencia de la participación en la agenda educativa oficial alimenta su olvido o desconsideración.

En muy pocas ocasiones se escuchó en los discursos referencias directas al currículum explícito, aun cuando la tradición pedagógica nos indica diferentes estrategias para fomentar la participación a este nivel. La elección de contenidos por parte del alumnado aparece en el desarrollo de proyectos, como metodología que incorpora la voz del alumnado en la dimensión curricular. Esta metodología parte de una visión del aprendizaje como investigación, con distintos grados de autonomía de los alumnos, en la elección de las temáticas y de los procesos más pertinentes para la recolección y procesamiento de la información y producción. Este método puede ejemplificar la necesidad de un planteamiento docente, desde la planificación, del grado de implicación que se espera del alumnado. 
Entre las experiencias más generalizadas encontramos como objeto de participación de forma predominante la intervención en la comunidad. Una de las propuestas más generalizadas consiste en la realización de jornadas temáticas como reunión de los distintos agentes educativos en "el día de..." (la multiculturalidad, la convivencia, la paz, la solidaridad...) con un carácter eminentemente social, festivo y cultural. En ellas se sitúa el foco en un proyecto anual del que la jornada solamente es su culminación y síntesis, que permite trasladar a las familias los resultados de las tareas realizadas, su sensibilización e implicación en la temática elegida.

"[...] ese día era como el broche final al proyecto que habiamos empezado en septiembre con el tema de la convivencia, con el que pretendimos y conseguimos solucionar los problemas de convivencia que se daban en el patio" GD de Profesionales de la Educación

También se explica alguna actividad intercentros donde en representación de sus grupos escolares un grupo de alumnos se reúne para compartir y sintetizar sus propuestas, aunque el proceso que posteriormente se sigue con ellas, para darles publicidad, hacer el seguimiento de su desarrollo y ejecución y para su evaluación final no parece suficientemente claro.

"Trabajamos en los tres colegios de una manera coordinada una vez al mes, desde enero, hacemos nuestras propuestas, que son sus propuestas, mezclando a los críos de los colegios en grupos heterogéneos [...] alli se hacen las propuestas, que son a su vez lo que han traído de sus clases" GD Profesionales de la Educación: Escuelas enredadas

\section{¿En qué escenarios surgen las principales posibilidades para la participación?}

El escenario predominante de participación es el aula, donde transcurre la mayor parte del tiempo escolar, como espacio de convivencia y de experiencias interpersonales. Es el lugar donde el alumnado puede expresar más fácilmente su punto de vista desde la cotidianeidad. Aun siendo interesantes estas prácticas de diálogo y debate no queda claro cómo se materializa esa participación de forma que niños y niñas pueden reconocer, finalmente, cambios como resultados visibles y reconocibles producto de sus aportaciones.

El centro también es el punto de partida de algunas actividades que están recogidas en el Proyecto Educativo y que se articulan en los distintos grupos o, de forma más extraordinaria como expusimos anteriormente, en una jornada temática. Pero este tipo de participación suele ser muy puntual.

Una mayoría de iniciativas siguen la dirección de arriba a abajo; es decir con escasa presencia de prácticas originadas en el aula, de abajo a arriba. No se registraron iniciativas surgidas de los grupos de alumnos que alcanzaran un desarrollo de toda la comunidad. Esto pone de relieve como la participación infantil se aborda desde una óptica del alumnado como objeto de derechos en la que la persona adulta decide el cómo y el cuándo puede ejercerla (Veenhoven, 1996). Desde el paradigma de la infancia como "sujeto de derechos" habrían de proporcionarse 
escenarios y experiencias en que fuera posible realizar propuestas y desarrollar iniciativas.

\section{De la participación anecdótica hacia la participación real}

Uno de los temas más relevantes fue el grado de su participación dada la discrepancia clara entre la percepción de los adultos y la percepción del alumnado.

De forma general, las experiencias que se han recogido en los grupos de discusión consistían en prácticas de intercambio oral y debates para hacer consultas. Sin embargo, la percepción de las niñas y los niños es que estas consultas no son de participación real, fundamentalmente porque los efectos reales son muy pocos y las acciones e innovaciones sobre las que fueron consultados no son vistos como una realidad. La expresión del alumnado en los grupos de discusión sobre su implicación en experiencias de centro hace referencia a la demanda de opinión, en dinámicas de asamblea o debate, en el tratamiento de un conflicto o temática y la generación de propuestas para su resolución, pero sin identificar su contribución en las decisiones finalmente tomadas.

"No. Solamente nos preguntan qué queremos hacer, si queremos ir aquí o alli, pero después hacemos otra cosa. Hacemos debates en clase, tratamos temas, pero no participamos [...]" GD de Niñas y Niños

De acuerdo con esto, llama la atención, como actividades realizadas en la escuela y definidas como participativas por docentes, madres y padres son omitidas en los discursos del alumnado. $\mathrm{Su}$ participación podría tratarse de una adhesión o consentimiento a las propuestas de los adultos. En alguna ocasión las manifestaciones de los docentes dejan entrever esa confusión, extrayendo del discurso del alumnado algunas ideas que después los profesores cogemos y [a las que] damos forma:

"En junio, siempre a final de curso, los niños se reúnen por clases, en asamblea, y hacen propuestas de qué quieren, qué es lo más importante que quieren hacer el curso siguiente. Y entonces, estas aportaciones seo debaten en la asamblea, los profesores sacamos algunas ideas que después cogemos y [a las que] damos forma" GD de Profesionales de la Educación

Desde los distintos referentes propuestos para conocer el grado de participación del alumnado (Brown, 2001; Fielding y McGregor, 2005; Fielding, 2001; Hart, 1992; Martínez Rodríguez, 2010; Robinson y Fielding, 2007; Shier, 2001) interpretamos que, en general, excluyendo algunas prácticas de participación genuina, las experiencias que se narran corresponden a los niveles más bajos de las escalas propuestas por los distintos autores.

Podríamos encontrar como argumentos explicativos, entre otros, la ausencia de cultura participativa y la concepción social de la infancia como etapa sujeta a protección y provisión, pero sin un auténtico reconocimiento como sujetos activos de la sociedad. Es decir, sin capacidad para interesarse de forma responsable en las cuestiones de interés comunitario que les afectan, ni de las de su propio desarrollo. La 
práctica, pero, parece contradecirse con las aspiraciones de algunos profesionales que abogan por dinámicas de mayor implicación del alumnado.

"Una de las primeras cosas que les tenemos que enseñar a nuestros alumnos es a saber que son seres, personas libres y con capacidades para poderse desarrollar en una sociedad en libertad y democráticamente. Y evidentemente una de las herramientas es la participación. Tiene que ir aprendiéndolo y ejercitándolo desde la escuela [...]" GD de Profesionales de la Educación

\section{Facilitar la participación a través del formato}

El formato de participación más común que se presenta es la expresión de la opinión y anhelos del alumnado en procesos deliberativos en el aula, utilizando predominantemente el lenguaje oral. Las asambleas o debates suelen tener una relación directa con hechos sucedidos, sirviendo para su valoración, o para la planificación de algún evento próximo. La supremacía del lenguaje oral, es comprendida por algunos como una limitación para una parte importante del alumnado que quizá se expresaría más ampliamente mediante otros sistemas, como propone Greene (2005), que permitirían promover un diálogo más amplio desde la creatividad. Un segundo formato, con una presencia significativa, es el de acción o de actuación donde los alumnos son actores, con una actuación intelectual y motriz generalmente realizando aquello que se les encargó con una cierta responsabilidad.

\section{Conclusiones y propuestas}

El análisis de los discursos de los grupos de discusión que se desarrollaron en la investigación, en relación a las experiencias del alumnado en la vida escolar, permite, conocer las experiencias y los niveles de participación (objetivo 1), que constatan niveles situados en la parte baja de las escalas estudiadas. A la vez, en la identificación de barreras de la organización escolar en relación a la participación (objetivo 2) se evidencia la ausencia general en los centros educativos de un consenso conceptual sobre laparticipación: ¿de qué participación hablamos cuando hablamos de participación? Ambas constataciones son coherentes con una educación fuertemente adherida a las tradiciones, en la que la infancia se representa como etapa donde los sujetos carecen de la capacidad para tomar decisiones sobre aquello que afecta a sus vidas. El mismo alumnado no reconoce ni su opinión ni sus propuestas en las iniciativas que el centro impulsa.

A las carencias conceptuales podemos asociar otras de naturaleza pedagógica, relacionadas con las metodologías, las estrategias dialógicas, la negociación, entre otras, como modelos de interacción útiles para desarrollar hábitos y prácticas de participación. Estas circunstancias, en tanto que obstáculos, deberían interpretarse como espacios susceptibles de mejora, como ámbitos de formación docente para promover una educación en la autonomía, la autorregulación y la responsabilidad en educación infantil, primaria y secundaria. Poner en valor la participación de la infancia y la concepción de los niños y niñas como sujetos activos de derechos requiere 
diferentes niveles y formas de actuaciónque abarcan la vida del aula, la vida y organización del centro, así como la participación en el entorno social y ciudadano del alumnado. Ello requiere una formación metodológica que ofrezca a los docentes nuevas formas de pensar en los procesos de enseñanza-aprendizaje, así como nuevas fórmulas para incorporar al alumnado en los procesos de la vida de los centros. La adhesión de nuestro país a la Convención de los Derechos del Niño justifica la conveniencia de su incorporación a la formación inicial y continúa de los profesionales de la educación, por su carácter de ineludible cumplimiento.

Algunas de las experiencias planteadas muestran la escuela como el espacio social y educativo más adecuado para experimentar la participación como forma de compromiso de los alumnos con ellos mismos y los demás. La vivencia de la democracia favorece la incorporación de la cultura dialógica para la regulación de la convivencia y en el funcionamiento del centro. En estas prácticas se observan dos grandes campos de actuación: el curricular y el institucional. La experiencia de la participación potencia la eficacia educativa porque la socialización y la ciudadanía forman parte del currículum en cualquiera de las etapas escolares y, a la vez, contribuye a una convivencia favorable para la adquisición de las competencias curriculares. En definitiva, la participación puede comprenderse desde su potencialidad de mejora institucional por su relación con el aprendizaje y el desarrollo de la organización. La experiencia y resultados de UNICEF UK (2010) evidencian como el alumnado puede analizar su realidad más inmediata desde la CDN, como referente universal para incidir sobre ella, favoreciendo el conocimiento de derechos y responsabilidades.

Superar el discurso oculto por confuso es otra de las ideas que aparece en la diversidad de expresiones en este estudio. La demanda de explicitar un relato preciso sobre la participación es compartida por gran parte de los profesionales, para ir más allá de la visión anecdótica de "el día de". El desconocimiento de la CDN y la ausencia del consenso sobre la idea de participación acaban suponiendo prácticas dispares y confusas en el mismo centro. Esto hace necesario cuestionar el día a día para: en primer lugar, desvelar conjuntamente lo tácito, las incoherencias entre lo que se dice, se hace y debería hacerse; para acordar una acción educativa común. Aunque en la mayoría de proyectos existe una mención a la implicación y corresponsabilización de la comunidad educativa, algunos docentes observan en la programación anual una ausencia clamorosa de concreciones en actividades de aula y centro. 


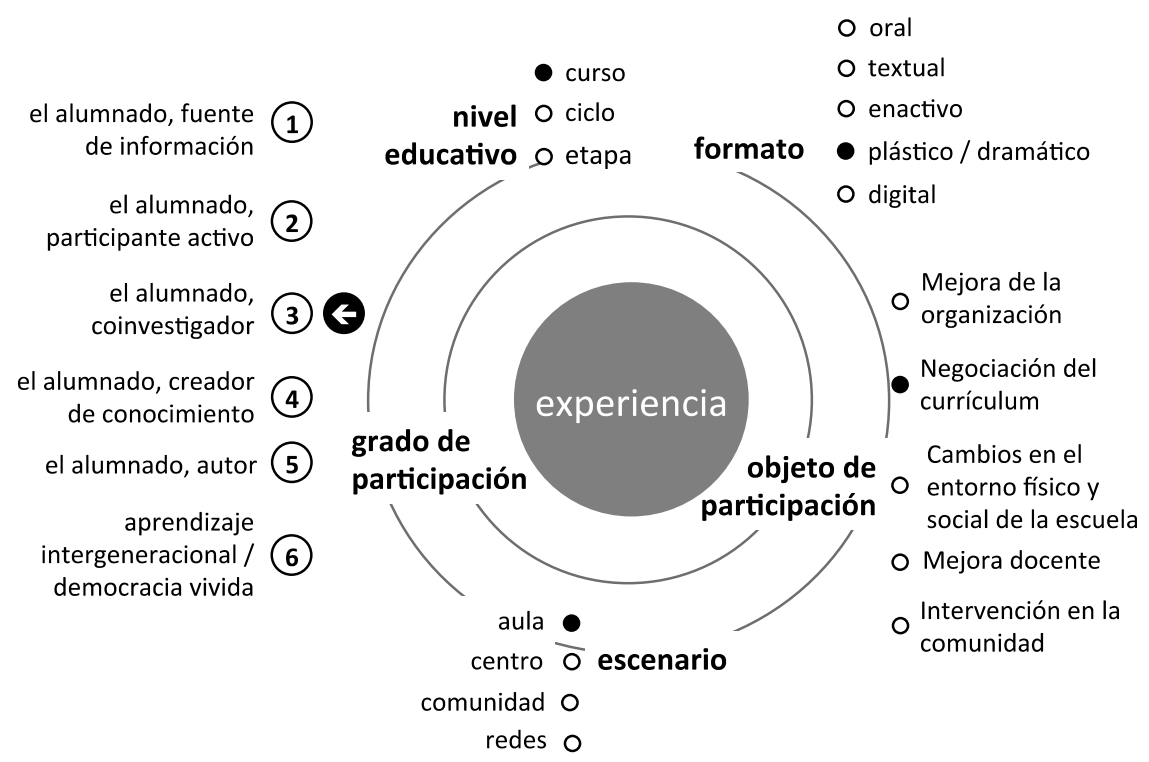

Gráfico 1: Dimensiones de análisis de la participación del alumnado en la escuela

Creemos necesario establecer distintos momentos para la revisión y de evaluación de la participación del alumnado. En una propuesta de síntesis desde la revisión realizada destacamos cinco dimensiones de análisis de la experiencia de participación (Gráfico 1), y tres momentos clave: a) al inicio de un proyecto de participación; b) en su planificación a largo o medio plazo y su concreción en un curso y c) en la evaluación del proyecto una vez implementado. Al inicio para conocer el punto de partida y poder establecer objetivos factibles que posibiliten un progreso real. La revisión de las prácticas a nivel de centro, etapa y nivel formarían parte de la formación conjunta, del establecimiento de una semántica común para la concreción del proyecto, donde las actividades puedan ser argumentadas en relación a las distintas dimensiones de análisis de la participación. En la planificación de los proyectos anuales, un análisis previo a su aprobación permitiría reconocer ocasiones, modalidad y grado donde explícitamente se contempla la implicación del alumnado. Este análisis permite planificar la participación en los distintos escenarios así como la dimensión(es) afectada(s), curricular e institucional.

La constatación de las diferencias entre las aspiraciones del centro y las realizaciones parece el camino oportuno en la concreción de nuevas propuestas para el ejercicio real de los derechos de la infancia. La organización escolar tiene, desde las expresiones recogidas, una potencialidad substancial para la experiencia de la participación. El consenso de la comunidad sobre una mayor implicación del alumnado en aquello que concierne a su vida y escolaridad es un reto para la formación de la ciudadanía, como compromiso y sentido de pertenencia a la sociedad democrática. 


\section{Referencias bibliográficas}

ALSINET, C. (2000). El Benestar en la infància. Lleida: Pagès.

BALSElls, M. À., COIDURAS, J., ALSINET, C., \& URREA, A. (2012). Derechos de la Infancia y Educación para el Desarrollo. Análisis de necesidades del sistema educativo. Lleida.

BlANCHET, A., \& GOTMAN, A. (2005). L'enquête et ses méthodes: l'entretien. Paris: Armand Colin.

BOLÍVAR, A. (1999). Cómo mejorar los centros educativos. Madrid: Síntesis.

BROWN, N. (2001, May). Focus on... a school working with Project 2. Communicating... The ESRC Network Projecte Newsletter, 1-4.

BRUNER, J. S. (2013). La educación, puerta de la cultura. Boadilla del Monte: Machado Grupo de Distribución.

CASAS, F., GONZÁlEZ, M., MONTSERRAT, C., NAVARRO, D., MALO, S., FIGUER, C., \& BERTRÁN, I. (2008). Informe sobre experiencias de participación social efectiva de niños, niñas y adolescentes. Madrid: Ministerio de Educación Política Social y Deporte.

CEBALlOS, N., \& SUSINOS, T. (2012). Reconociendo la voz del alumnado como medio de construcció de escuelas democráticas e inclusivas: análisis de experiencias. Quaderns Digitals, 71.

CONNELL, R. W. (1997). Escuelas y justicia social. Madrid: Morata.

COTS, J. (2005). El derecho a la participación de los niños. Revista de Educación Social, 4.

DURKHEIM, E., SCHNURER, H., \& WILSON, E. K. (1961). Moral Education: A Study in the Theory and Application of the Sociology of Education. New York: Free Press of Glencoe.

LEY ORGÁNICA 8/1985, de 3 de julio, Reguladora del Derecho a la Educación (LODE). Boletín Oficial del Estado (España), 4 de julio de 1985, 159, 21015-21022.

FALLERY, B., \& RODHAIN, F. (2007). Quatre approches pour l'analyse de données textuelles: lexicale, linguistique, cognitive, thématique. In XVIème Conférence Internationale de Management Stratégique. Montréal, 6-9 Juin (pp. 1-27). Montréal: Université du Québec.

FIELDING, M. (2001). Students as radical agents of change. Journal of Educational Change, 2, 123-141.

FIELDING, M. (2004). Transformative approaches to student voice: theoretical underpinnings, recalcitrant realities. British Educational Research Journal, 30(2), 295-311. doi:10.1080/0141192042000195236 
FIELDING, M. (2011). La voz del alumnado y la inclusión educativa: una aproximación democrática radical para el aprendizaje intergeneracional. Revista Interuniversitaria de Formación de Profesorado, 25(1), 31-61.

FIELDING, M. (2012). Beyond Student Voice: Patterns of Partnership and the Demands of Deep Democracy. Revista de Educación, 359(Septiembre-diciembre), 45-65. doi:10.4438/1988-592X-RE-2012-359-195

FIELDING, M., \& BRAGG, S. (2003). Students as Researchers: Making a difference. Cambridge: Pearson Publishing.

FIELDING, M., \& MCGREGOR, J. (2005). Deconstructing student voice: new spaces for dialogue or new opportunities for surveillance? In Speaking up and speaking out: International perspectives on the democratic possibilities of student voice. Montréal: AERA Montreal.

FUENTES-PELÁEZ, N. (2011). Escoltar la veu dels infants i joves: una via de lluita contra l'exclusió social dels joves del quart món. In M. A. Balsells (Ed.), Quart món i infància. Conceptes, reptes i oportunitats (pp. 99-122). Barcelona: Icaria.

FULLAN, M. (1993). Change Forces. The dots of educational reform. Londres: The Falmer.

GREENE, M. (2005). Liberar la imaginación: ensayos sobre educación, arte y cambio social. Barcelona: Graó.

HARGREAVES, A., EARL, L. M., \& RYAN, J. (1998). Una educación para el cambio: reinventar la educación de los adolescentes. Madrid: Octaedro.

HART, R. A. (1992). Children's participation from tokenism to citizenship. Florence, Italy: UNICEF Innocenti Research Centre.

JIMÉNEZ-TRENS, M. A., \& NAVARIDAS, F. (2012). Cómo son y qué hacen los "maestros excelentes": la opinión de los estudiantes. Revista Complutense de Educación, 23(2), 463-485. doi:10.5209/rev_RCED.2012.v23.n2.40038

KAUFMANN, J.-C. (2011). L'enquête et ses méthodes. L'entretien compréhensif. Paris: Armand Colin.

KOHLBERG, L., POWER, F. C., \& HIGGINS, A. (1997). La Educación moral según Lawrence Kohlberg. Barcelona: Gedisa.

LODGE, C. (2005). From hearing voices to engaging in dialogue: problematising student participation in school improvement. Journal of Educational Change, 6(2), 125-146. doi:10.1007/s10833-005-1299-3

MARTÍN DEL POZO, R., \& DE JUANAS OLIVA, Á. (2013). La valoración de los maestros sobre la utilización didáctica de las ideas de los alumnos. Revista Complutense de Educación, 24(2), 267-285. doi:10.5209/rev_RCED.2013.v24.n2.42079 
MARTÍNEZ RODRÍGUEZ, J. B. (2010). El currículo como espacio de participación. La democracia escolar ¿es posible? In J. Gimeno Sacristán (Ed.), Saberes e incertidumbres sobre el currículum (pp. 162-179). Madrid: Morata.

MAYOR-ZARAGOZA, F. (1989). Mañana siempre es tarde. Madrid: Espasa Libros.

MITRA, D. L., SERRIERE, S., \& KIRSHNER, B. (2013). Youth Participation in U.S. Contexts: Student Voice Without a National Mandate. Children \& Society, Online. doi:10.1111/chso.12005

PULIDO VALERO, R., CALDERÓN LÓPEZ, S., MARTÍN-SEOANE, G., \& LUCAS-MOLINA, B. (2014). Implementación de un programa de mediación escolar: Análisis de las dificultades percibidas y propuestas de mejora. Revista Complutense de Educación, 25(2), 375-392. doi:10.5209/rev_RCED.2014.v25.n2.41610

ROBINSON, C., \& FIELDING, M. (2007). Children and their primary schools: Pupil's voices. Cambridge.

RODRÍGUEZ SEDANO, A., \& AGUILERA, J. C. (2009). De la constitución moral de la sociedad a la educación moral según Durkheim. Revista Española de Pedagogía, 243(mayo-agosto), 319-336.

RUDDUCK, J. (2007). Student Voice, Student Engagement, and School Reform. In D. Thiessen \& A. Cook-Sather (Eds.), International Handbook of Student Experience in Elementary and Secondary School (pp. 587-610). Dordrecht: Springer.

RUDDUCK, J., \& FLUTTER, J. (2007). Cómo mejorar tu centro escolar dando la voz al alumnado. Madrid: Morata.

SEBBA, J., \& ROBINSON, C. (2010). Evaluation of UNICEF UK's Rights Respecting Schools Award. London.

SHIER, H. (2001). Pathways to Participation: Openings, Opportunities and Obligations. Children \& Society, 15(2), 107-117. doi:10.1002/CHI.617

SMYTH, J. (2007). Toward the Pedagogically Engaged School: Listening to Student Voice as a Positive Response to Disengagement and "Dropping Out"? In D. Thiessen \& A. Cook-Sather (Eds.), International Handbook of Student Experience in Elementary and Secondary School (pp. 635-658). Dordrecht: Springer. doi:10.1007/1-4020-3367-2_25

SUSINOS, T., \& CEBALLOS, N. (2012). Voz del alumnado y presencia participativa en la vida escolar. Apuntes para una cartografía de la voz del alumnado en la mejora educativa Student Voice and Participatory Presence in School Life. Notes for Mapping Student Voice in Educational Improvement. Revista de Educación, 359(septiembre-diciembre), 24-44. doi:10.4438/1988-592X-RE-2012-359-194

TONUCCI, F. (1998). La ciudad de los niños: un modo nuevo de pensar la ciudad. Madrid: Fund. Germán Sánchez Ruipérez. 
TORRES SANTOMÉ, J. (2010). La justicia curricular. El caballo de Troya de la cultura escolar. Madrid: Morata.

TRILLA, J., \& NOVELLA, A. M. (2011). Participación, democracia y formación para la ciudadanía. Los consejos de infancia. Revista de Educación, 356(septiembrediciembre), 23-43.

UNICEF. (2008). Rights Respecting Schools in England. Briefing Paper. London: UNICEF-UK.

VEENHOVEN, R. (1996). Developments in satisfaction research. Social Indicators Research, 37, 1-46.

WEST, M., \& HOPKINS, D. (2003). Reconceptualizing School Effectiveness and Improvement. In B. Davies \& J. West-Burnham (Eds.), Handbook of Educational Leadership and Management. London: Perason Longman.

\section{Correspondencia con los autores}

\section{Jordi L. COIDURAS RODRÍGUEZ}

Facultad de Educación, Psicología y Trabajo Social

Universidad de Lleida.

Avda. de l'Estudi General, 4

25001 Lleida

e-mail: coiduras@pip.udl.cat

\section{M. Àngels BALSELLS BAILÓN}

Facultad de Educación, Psicología y Trabajo Social

Universidad de Lleida.

Avda. de l'Estudi General, 4

25001 Lleida

e-mail: balsells@pip.udl.cat

\section{Carles ALSINET MORA}

Facultad de Educación, Psicología y Trabajo Social

Universidad de Lleida. Avda. de l'Estudi General, 4

25001 Lleida,

e-mail: alsinet@pip.udl.cat

\section{Aida URREA MONCLÚS}

Facultad de Educación, Psicología y Trabajo Social

Universidad de Lleida.

Avda. de l'Estudi General, 4

25001 Lleida,

e-mail: aurrea@pip.udl.cat 
Ignacio GUADIX GARCÍA

UNICEF-Comité Español.

C/ Mauricio Legendre, 36

28046 Madrid,

e-mail: nguadix@unicef.es

Oscar BELMONTE CASTELL

UNICEF-Comité Español.

C/ Mauricio Legendre, 36

28046 Madrid,

e-mail: obelmonte@unicef.es 